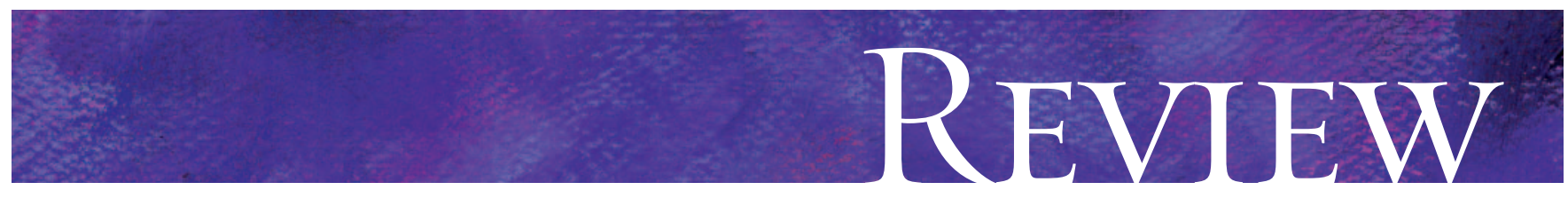

\title{
Sleep and aging: 2. Management of sleep disorders in older people
}

\author{
Norman Wolkove, Osama Elkholy, Marc Baltzan, Mark Palayew
}

\section{ABSTRACT}

The treatment of sleep-related illness in older patients must be undertaken with an appreciation of the physiologic changes associated with aging. Insomnia is common among older people. When it occurs secondary to another medical condition, treatment of the underlying disorder is imperative. Benzodiazepines, although potentially effective, must be used with care and in conservative doses. Daytime sedation, a common side effect, may limit use of benzodiazepines. Newer non-benzodiazepine drugs appear to be promising. Rapid eye movement (REM) sleep behaviour disorder can be treated with clonazepam, levodopa-carbidopa or newer dopaminergic agents such as pramipexole. Sleep hygiene is important to patients with narcolepsy. Excessive daytime sleepiness can be treated with central stimulants; cataplexy may be improved with an antidepressant. Restless legs syndrome and periodic leg-movement disorder are treated with benzodiazepines or dopaminergic agents such as levodopa-carbidopa and, more recently, newer dopamine agonists. Treatment of obstructive sleep apnea includes weight reduction and proper sleep positioning (on one's side), but may frequently necessitate the use of a continuous positive air-pressure (CPAP) device. When used regularly, CPAP machines are very effective in reducing daytime fatigue and the sequelae of untreated obstructive sleep apnea.

CMAJ 2007;176(10):1449-54

I $\mathrm{n}$ part $\mathrm{I}$ of this review we summarized the changes in sleep that occur with aging. ${ }^{1}$ We also reviewed several of the more common sleep disorders seen among older people. In this paper, we will attempt to highlight the management of these conditions.

\section{Causes of secondary insomnia}

Insomnia, particularly sleep disruption, is common among $\omega$ older people because coexisting medical conditions that cause sleepiness are so frequent in this subpopulation. In these cases, treatment must be targeted at the illness in question, sometimes with specific management choices designed to improve sleep efficiency. Pain related to musculoskeletal disorders, including arthritis, constitutes one of the most common causes of insomnia in this subpopulation. In addi- tion to anti-inflammatory medication, bedtime acetaminophen often promotes more restful sleep. Nocturia is also a common cause of sleep loss (and therefore, daytime fatigue) in elderly patients. As well as interrupting sleep, nocturia is a primary contributor to nocturnal falls and related hip fractures. ${ }^{2}$ When present, nocturia should prompt the search for sleep apnea, diabetes, prostatic disease (in men) and bladder prolapse (in women), and appropriate treatment thereof. Patients with obstructive lung disease may benefit from the use of a long-acting $\beta$ agonist before bedtime, to minimize disruptive nocturnal dyspnea. Gastrointestinal reflux that distubs sleep should prompt dietary counselling and may require therapy, such as use of a proton pump inhibitor. Aggressive treatment of congestive heart failure will improve the symptoms of paroxysmal nocturnal dyspnea and orthopnea, and enhance sleep quality.

Insomnia may be the presenting complaint in older patients who have underlying depression or early dementia. These illnesses are less obvious and require more time for proper assessment and treatment. Depressed patients who have insomnia will often improve with antidepressant medication in doses appropriate for the older patient. ${ }^{3}$ In those with dementia who exhibit agitated evening or nighttime behaviour (sundowning), simple behavioural and environmental measures can be useful: reassurance, late-day limitation of extraneous stimuli, and a familiar, restful environment that is not totally dark. Ultimately, however, pharmacologic intervention is often necessary. ${ }^{4}$

Since older patients are the greatest consumers of medications, a thorough drug history is important. Consider over-the-counter medications, as well; these often contain ingredients that interfere with sleep (e.g., caffeine). Whenever possible, medications that may interfere with sleep should be eliminated; examples can be found in Table 2 of part I of this review. ${ }^{1}$

\section{Primary insomnia}

Primary insomnia is defined as sleeplessness that is not attributable to a medical, psychiatric or environmental cause. ${ }^{5}$ Treatment of primary insomnia in older patients should take into account the psychological changes in sleep associated with age, described in part I of this review. In most cases, the initial approach should be behaviour modification. ${ }^{6}$ Box I summarizes the general principles of sleep hygiene that are applicable. 
Phototherapy is an interesting nonpharmacological therapy for insomnia. As already described, older people often have a phase advance in their circadian rhythm that leads to earlier sleep onset and earlier, often nighttime, awakening. Evening light therapy appears to be a particularly effective treatment for early-morning insomnia from a phase-advanced circadian rhythm. ${ }^{7}$ Timed exposure to bright light has improved sleep efficiency and increased total sleep time, rapid-eye-movement (REM) sleep and slow-wave sleep in older people. ${ }^{8}$ Light therapy may be effective even when given earlier in the day: bright light exposure at lunchtime improved disturbed sleep in nondemented residents of a geriatric facility. ${ }^{9}$ In patients with dementia, bright light therapy was also effective in reducing daytime sleep..$^{10}$ More specifically, evening light exposure has been shown to ameliorate disturbances of the sleep-wake cycle in some patients with Alzheimer's disease. ${ }^{11}$

Although the mechanism by which light exposure improves sleep is not entirely clear, the implications, especially for people in institutions, are obvious. During the daytime, a well-lit, stimulating environment with exposure to natural light should be the goal in all long-term residential facilities.

Benzodiazepines have been the most common hypnotics used by older patients. They can be divided roughly into 3 groups: long-acting, intermediate-acting and short-acting (Table I). Benzodiazepines suppress stages 3, 4 and REM sleep, and increase stage 2 sleep. Clinically, they decrease sleep latency and nocturnal awakenings. However, caution must be exercised when these drugs are prescribed for older patients. With advancing age, people become more sensitive to the effects of benzodiazepines on the central nervous system and (because of altered pharmacodynamics) more prone to side effects. In general, when administering benzodiazepines to elderly patients, adhere to the familiar admonition,
"Start low, go slow." Begin with no more than half the maximal dose recommended for younger adult patients, titrate slowly, and prescribe the drug for short periods only. Because continued use can produce drug tolerance, dependence and the potential for withdrawal symptoms, encourage patients to limit their use to 2 or 3 nights per week. Use of benzodiazepines by geriatric patients has been associated with mobility problems and decreased ability to perform the activities of daily living. ${ }^{12}$ Older patients taking these medications should be carefully monitored for daytime sedation and impaired motor coordination; they are at increased risk of falling, with resultant hip fracture. ${ }^{13}$

Other potential side effects in older patients include confusion, amnesia, night wandering, paradoxical agitation and various degrees of cognitive impairment. ${ }^{14}$ Older patients who have been taking benzodiazepines long-term are more likely to experience postoperative confusion. ${ }^{15}$ Because hypnotic agents, especially benzodiazepines, can contribute to upper-airway obstruction during sleep, avoid prescribing them for patients with known or suspected obstructive sleep apnea. Side effects in this age group are so common that Glass and colleagues, ${ }^{16}$ after a comprehensive analysis, concluded that the benefits of the drugs may not justify the increased risk in people over 6o years of age, especially if the patient has additional risk factors for adverse cognitive or psychomotor events (e.g., confusion, falls).

For sleep-onset insomnia, a short-acting agent such as triazolam or oxazepam may be effective. However, case reports of confusion, amnesia and behaviour problems with triazolam have been reported. ${ }^{6}$ In a patient with early-morning awakening, an intermediate agent such as temazepam may be more useful. Long-acting benzodiazepines such as diazepam, flurazepam and chlordiazepoxide are not recommended for

\section{Box 1: Fundamentals of good sleep hygiene}

What to do

- Use your bed only for sleep and sexual activities

- If you cannot sleep, get out of bed and read or do other relaxation activities before attempting to sleep again

- Make the quality of your sleep a priority

- Go to bed and get up at the same time every day

- Ensure a restful environment:

o A comfortable bed in a cool, well-ventilated room

o Protection from light and noise

- Develop and maintain bedtime "rituals" that make going to sleep a familiar routine; for example,

- Prepare for sleep with 20-30 minutes of relaxation (e.g., soft music, meditation, breathing exercises, yoga)

- Take a warm bath

- Have a light snack, which could include:

o Warm milk

o Foods high in tryptophan, such as bananas

o Carbohydrates, which can help induce sleep (whereas proteins promote wakefulness)
What to avoid

- In general, refrain from:

- Napping, especially after 3:00 pm

- Going to sleep too early in the evening (this can lead to phase advance syndrome)

- Before bedtime (or late in the day), avoid:

- Heavy eating

- Consumption of caffeine or alcohol

- Smoking (nicotine interferes with sleep)

- Exercise, which is a stimulant (although daytime activity will promote later sleep)

- While you try to fall asleep, avoid:

- Thinking about life issues

- Problem-solving

- Rehashing the events of the day 
elderly patients. Clonazepam should rarely be used, because of its potency and long duration of action.

New non-benzodiazepine agents are now becoming increasingly popular and have been shown to be effective in the short-term treatment of insomnia. They all share a relatively brief half-life, and therefore have less potential for residual daytime sedation. In addition, psychomotor performance and memory appear to be better preserved than they are with benzodiazepines. ${ }^{17}$ Non-benzodiazepine drugs generally disrupt normal sleep architecture less than do benzodiazepines. ${ }^{17} \mathrm{Ex}-$ amples of non-benzodiazepine agents include zolpidem, zaleplon, zopiclone and eszopiclone. ${ }^{18-20}$

Amitriptyline is sometimes prescribed as a sleep inducer, particularly when there is a suggestion of underlying depression. However, older patients are particularly sensitive to its anticholinergic side effects, including tachycardia, urinary retention, constipation, cognitive impairment, confusion, sedation and delirium. Curtis and associates, ${ }^{21}$ using criteria based on risk of adverse events, found amitriptyline to be one of the most commonly prescribed drugs that they concluded should be avoided for elderly patients. Trazodone, an antidepressant, has been used off-label, in smaller doses, by many physicians to treat insomnia. However, there is little objective data to support this use, and no randomized double-blind trials have been performed to assess its role in the treatment of primary insomnia. ${ }^{22}$ Evidence suggests that it may be efficacious when

Table 1: Sedative or hypnotic medications in common use

Drug Usual adult dose, ${ }^{*} \mathrm{mg}$

Benzodiazepines

Short-acting (half-life $<10 \mathrm{~h}$ )

Oxazepam

$15-30$

Triazolam

$0.125-0.25$

Intermediate-acting (half-life about 10-20 h)

Alprazolam

$0.25-0.5$

Estazolam (unavailable in Canada)

$0.5-2$

Lorazepam

$0.5-1$

Temazepam

15

Long-acting (half-life $>20 \mathrm{~h}$ )

Chlordiazepoxide

5

Clonazepam

$0.25-0.5$

Diazepam

2-10

Flurazepam

15-30

Non-benzodiazepines $†$

Eszopiclone

1-3

Ramelteon

8

Zaleplon

5-10

Zolpidem

5-10

Zopiclone†

5-7.5

*For elderly patients, start with about half the average adult starting dose and adjust if necessary.

tOf the non-benzodiazepines, only zopiclone is available in Canada (as of this writing). agitation or depression is contributing to insomnia, or where insomnia is caused by a stimulant antidepressant. ${ }^{23}$

Antihistamines with sedative properties, sometimes recommended to younger people, may cause excessive somnolence and confusion in older patients, and should be avoided.

Melatonin, a naturally occurring hormone produced by the pineal gland, is thought to be an endogenous sleep inducer. Its secretion is stimulated by darkness and inhibited by light. Melatonin concentrations in older patients peak later at night at about half the plasma levels of younger people. ${ }^{24}$ The relative melatonin deficiency that accompanies aging may therefore contribute to insomnia. Promoted by some as a more "natural" sleep remedy, some studies ${ }^{25}$ have in fact shown melatonin administration to be modestly effective in improving sleep quality in elderly patients, and reducing sundowning in those with Alzheimer's disease. However, Buscemi and coauthors, ${ }^{26,27}$ in their meta-analysis, concluded that melatonin, although safe, is of limited value in treating most primary or secondary sleep disorders. There was some evidence that short-term use of melatonin was useful in treating delayed sleep-phase syndrome, ${ }^{26}$ a disorder of sleep timing in which people are able to fall asleep only at late times, and then have difficulties waking up for morning activities. The authors cautioned that larger, randomized, controlled trials are necessary to yield evidence of effectiveness before widespread use can be advocated. ${ }^{26}$ Interestingly, ramelteon, a selective agonist for the melatonin receptors, has been approved for the treatment of insomnia in the United States. It has been shown to reduce sleep latency and increase total sleep time without significant adverse effects. ${ }^{28}$

\section{REM-sleep behaviour disorder}

The treatment of REM-sleep behaviour disorder should include a safe sleep environment, obtained by the removal of potentially dangerous objects from the bedroom. Both patient and bed partner should be educated in all aspects of the disorder, especially the potential for inadvertent self-harm at night. Removal of a medication that promotes REM-sleep behaviour disorder activity, such as selective serotonin-reuptake inhibitor (SSRI) antidepressants, may be beneficial. When associated with a neurodegenerative condition such as Parkinson's disease, multiple sclerosis or Alzheimer's dementia, treatment of the primary disorder, when possible, is appropriate. When drug treatment is thought to be warranted, clonazepam may be used and is effective. The initial dose is generally $0.5 \mathrm{mg}$ at bedtime, with some patients requiring up to I mg. Levodopa-carbidopa has been used in patients with REM-sleep behaviour disorder and early Parkinson's disease. Dopaminergic agents such as pramipexole have been found to be effective in this disorder and are now emerging as firstline therapy. ${ }^{29}$ Regardless of which treatment is used, longterm therapy is usually required because symptoms, once established, tend to persist.

\section{Narcolepsy and cataplexy}

Proper sleep hygiene is important in patients with narcolepsy. A regimen of 7-8 hours of sleep nightly in conjunction with 
scheduled daytime naps can improve symptoms. Pharmacological agents that act as central stimulants, such as methylphenidate and modafinil have been found to be effective. ${ }^{30}$ Tricyclic antidepressants have been used with success to decrease the frequency of cataplexy; however, their side effects (including dry mouth, blurred vision, difficulty urinating, constipation and orthostatic hypotension) may be particularly intolerable to older patients. The newer SSRIs may also be helpful in combination with stimulants for the treatment of narcolepsy with cataplexy. Sodium oxybate, which has been approved in the United States for the treatment of narcolepsy, ${ }^{31}$ can reduce the frequency of cataplexy attacks and improve daytime alertness. ${ }^{32}$ However, more studies are needed to assess its safety and efficacy in the older population.

\section{Sleep-related movement disorders}

Because the pathogenesis of restless legs syndrome and periodic leg-movement disorder is poorly understood, treatment is largely directed at symptom control. In general, the approach to treatment of these 2 disorders has considerable overlap (Table 3 of part $\mathrm{I}^{1}$ ). Caffeine-containing foods and beverages that can exacerbate symptoms should be reduced or eliminated. Medications known to aggravate symptoms of restless legs syndrome (e.g., calcium-channel blockers, metoclopramide, antihistamines, phenytoin, SSRIs) should be avoided, if possible. ${ }^{33}$ Iron supplements are indicated if test results for ferritin are less than $50 \mu \mathrm{g} / \mathrm{L} .^{33}$

Historically, both of these disorders have been treated with benzodiazepines. Clonazepam in a dose of $0.5-1.0 \mathrm{mg}$ at bedtime is frequently employed, which may be sufficient to reduce symptoms to more tolerable levels. Even when periodic leg movements continue, this treatment may enable the patient to sleep without waking, despite the motor activity. Again, benefit must be weighed against risk, including (in elderly people) the potential for daytime sedation, falls, confusion and worsening of sleep-related respiratory disorders.

Dopaminergic agents are widely used for treatment of these movement disorders. ${ }^{33,34}$ Symptom control occurs at a lower dose than typically required for Parkinson's disease. Levodopa-carbidopa is administered at bedtime with a starting dose of $100 \mathrm{mg} / 25 \mathrm{mg}$, and increased if needed. Rebound symptoms can occur as the drug concentration drops. Repeat dosing in the middle of the night may be necessary; sustained-release preparations may therefore be more practical. Specific concerns with these medications include frequent "augmentation," that is, an increased tendency for symptoms of restless legs syndrome to occur in the early hours of the morning or afternoon rather than at night. ${ }^{33,34}$ Older patients are prone to daytime somnolence with dopaminergic agents, a side effect that may preclude or limit their use. Tolerance occurs, requiring increased dosages. Medication may need to be suspended for several months; reintroduction of treatment thereafter usually proves efficacious again. Elderly patients, especially those with Parkinson's disease or underlying dementia, are at risk of nocturnal hallucinations and sleep attacks when treated with dopaminergic medication at high doses.
Clinical studies have shown that the newer dopamine agonists pergolide, pramipexole and ropinirole are effective in relieving symptoms of restless legs and reducing periodic leg movements. ${ }^{35}$ Trenkwalder and coauthors ${ }^{36}$ showed that pergolide substantially reduced periodic movements and subjective sleep disturbances in patients with restless legs syndrome, and that the benefit persisted for (at least) a year. However, pergolide was recently withdrawn from the market in the United States because of an association with heart-valve abnormalities. ${ }^{37,38}$ Pramipexole has proven efficacious in the treatment of restless legs syndrome, with no decrease in therapeutic benefit even after almost 8 months of use. ${ }^{39}$ Recently, in a large, randomized, double-blind study, ${ }^{40}$ ropinirole improved symptoms of restless legs syndrome and was well tolerated. Pramipexole and ropinirole appear to be safe for older patients, and may be particularly useful to those at risk of, or who have experienced, side effects from levadopa-carbadopa ${ }^{41}$ Tolerance does not often develop with these newer drugs, unlike with levadopa-carbidopa. Daytime somnolence can occur, especially in older patients with Parkinson's disease. ${ }^{41}$ Since side effects of all dopamine agonists are doserelated, conservative dosage should be the rule.

Opioids are the oldest treatment for restless legs syndrome. Their method of action in controlling symptoms is uncertain. Such drugs are less well suited to geriatric patients because of a propensity to cause confusion, sedation and constipation. Use of opioids is therefore restricted to severe cases refractory to other forms of treatment, and those associated with chronic neuropathic pain.

Other medications that are occasionally tried as secondline agents include the anticonvulsants carbamazepine, gabapentin and the muscle relaxant baclofen. These are not often prescribed for elderly patients, however, because of their sedative properties.

\section{Snoring and obstructive sleep apnea}

A wide variety of products and devices have been advertised in the lay press and on the Internet as potential "cures" for chronic snoring. Most have little or no scientific support. General measures such as weight reduction, smoking cessation and abstinence from alcohol are usually recommended. Keeping the nasal passages clear by use of a humidifier or nasal steroids may be helpful in some cases. Snoring tends to occur more when someone sleeps on the back, so patients should be counselled to sleep on their side.

Generally, surgical options, which are currently gaining popularity, should be considered with caution for older patients. Occasionally, surgical treatment of upper-airway abnormalities such as a deviated septum, enlarged tonsils or nasal polyps may correct an isolated problem. Uvulopalatopharyngoplasty in theory opens the airway and tightens the tissues of the throat and palate, thereby facilitating unobstructed airflow. Laser-assisted uvulopalatoplasty is a technique by which a carbon dioxide laser is used to reshape and reduce the size of the uvula and superficial palatial tissue to create a noncollapsible, patent oropharyngeal airway. ${ }^{42}$ These procedures may improve snoring but will generally not com- 
pletely resolve any associated obstructive sleep apnea. Moreover, patients should be cautioned that surgical procedures for snoring have considerable postoperative morbidity in older patients. ${ }^{43}$ They may also worsen the severity of obstructive sleep apnea and make subsequent therapy with a continuous positive airway pressure (CPAP) device more difficult.

The treatment of obstructive sleep apnea includes the conservative measures already outlined for snoring. For clinically significant, symptomatic obstructive sleep apnea, the most common treatment is continuous positive airway pressure delivered by a compact bedside machine, usually through a nasal mask (nCPAP). ${ }^{44,45}$ By adjusting the applied pressure appropriately, the airflow acts as a pneumatic splint, keeping the pharyngeal airway open and preventing obstructive apnea. This may be of great benefit to symptomatic patients. When CPAP is used diligently, patients report improved sleep quality, daytime alertness and energy. ${ }^{46}$ However, many people, especially the elderly, have difficulty accepting the nightly ritual of CPAP use. Elderly men with benign prostatic hyperplasia and nocturia find it difficult to repeatedly reapply the mask and may therefore abandon its use. ${ }^{47}$ In general, compliance with CPAP can be improved by having a nurse or technician spend time teaching the patient the optimal use of the equipment and ensuring that a comfortable, well-fitted mask is chosen. ${ }^{46}$ One difficulty with CPAP treatment that can be particularly important to elderly patients is the cost of the machine. Some Canadian provinces reimburse all or part of this cost. As of December 2006, such a device costs about \$1500$\$ 2000$ in Montréal and is not covered by Quebec Medicare. Patients on a fixed income may find the cost prohibitive.

Finally, oral appliances may be successful in treating obstructive sleep apnea in certain well-chosen patients, but are often difficult for older patients to tolerate. ${ }^{48}$

\section{Central sleep apnea}

When the major cause of sleep disturbance is central sleep apnea, treatment options include nocturnal oxygen, CPAP or various other forms of mechanical ventilation. In a large Canadian study $y^{49}$ involving patients with central sleep apnea and congestive heart failure, where the mean age of participants was 63 years, CPAP treatment was found to reduce apnea events and improve oxygenation, ejection fraction and 6minute walk distance. However, a survival benefit was not demonstrated.

\section{Summary}

Sleep disturbances are common in elderly patients. Attention must be paid to the treatment of comorbid disease. Medications to treat insomnia, REM-sleep behaviour disorder, narcolepsy, restless legs syndrome and periodic leg-movement disorder may be highly efficacious; but to minimize side effects in this subpopulation, they must be administered judiciously and in conservative doses. CPAP is an effective method to treat obstructive sleep apnea and can greatly improve patients' quality of life. However, education and appropriate supervision are important to ensure adequate compliance.
This article has been peer reviewed.

From the Sleep Clinic, Mount Sinai Hospital Center, and the Faculty of Medicine, McGill University, Montréal, Que.

Competing interests: None declared.

Contributors: Norman Wolkove formulated the structure of the article. Osama Elkholy gathered information for the literature review. All authors contributed content, made critical revisions, and have seen and approved the final version for publication.

Acknowledgements: We thank Nathalie Bendavid and Karen Kennedy for their assistance in the preparation of this manuscript.

\section{REFERENCES}

I. Wolkove N, Elkholy O, Baltzan M, et al. Sleep and aging: 1. Sleep disorders commonly found in older people. CMAJ 2007;176(9):1299-304.

2. Stewart RB, Moore MT, May FE, et al. Nocturia: a risk factor for falls in the elderly. JAm Geriatr Soc 1992;40:1217-20.

3. Barkin RL, Schwer WA, Barkin SJ. Recognition and management of depression in primary care: a focus on the elderly: a pharmcotherapeutic overview of the selection process among the traditional and new antidepressants. Am J Ther 2000;7:205-26.

4. McGaffigan S, Bliwise DL. The treatment of sundowning: a selective review of pharmacological and nonpharmacological studies. Drugs Aging I997;10:I0-7.

5. Silber MH. Clinical practice. Chronic insomnia. N Engl J Med 2005;353:803-10.

6. Ringdahl EN, Pereira SL, Delzell JE Jr. Treatment of primary insomnia. J Am Board Fam Pract 2004;17:212-9.

7. Lack L, Wright $\mathrm{H}$, Kemp K, et al. The treatment of early morning awakening insomnia with 2 evenings of bright light. Sleep 2005;28:616-23.

8. Campbell SS, Dawson D, Anderson MW. Alleviation of sleep maintenance insomnia with timed exposure to bright light. J Am Geriatr Soc I993;4I:829-36.

9. Fukuda N, Kobayashi R, Kohsaka M, et al. Effects of bright light at lunchtime on sleep in patients in a geriatric hospital II. Psychiatry Clin Neurosci 200I;55:29I-3.

Io. Fetveit A, Bjorvatn B. Bright-light treatment reduces actigraphic-measured daytime sleep in nursing home patients with dementia: a pilot study. Am J Geriatr Psychiatry 2005;13:420-3.

II. Satlin A, Volicer L, Ross V, et al. Bright light treatment of behavioral and sleep disturbances in patients with Alzheimer's disease. Am J Psychiatry I992;I49:1028-32.

I2. Gray SL, LaCroix AZ, Hanlon JT, et al. Benzodiazepine use and physical disability in community-dwelling older adults. J Am Geriatr Soc 2006;54:224-30.

I3. Cumming RG, Le Couteur DG. Benzodiazepines and risk of hip fractures in older people: a review of the evidence. CNS Drugs 2003;17:825-37.

I4. Nelson J, Chouinard G; Canadian Society for Clinical Pharmacology. Guidelines for the clinical use of benzodiazepines: pharmacokinetics, dependency, rebound and withdrawal. Can J Clin Pharmacol I999;6:69-83

I5. Kudoh A, Takase H, Takahira Y, et al. Postoperative confusion increases in elderly long-term benzodiazepine users. Anesth Analg 2004;99:I674-8.

I6. Glass J, Lanctot KL, Herrmann N, et al. Sedative hypnotics in older people with insomnia: meta-analysis of risks and benefits. BMJ 2005;331:II69.

I7. Wagner J, Wagner ML. Non-benzodiazepines for the treatment of insomnia. Sleep Med Rev 2000;4:55I-8I.

I8. Sanger DJ. The pharmacology and mechanisms of action of new generation, nonbenzodiazepine hypnotic agents. CNS Drugs 2004;I8(Suppl I):9-I5 [discussion: 4I, 43-5].

I9. Cotroneo A, Gareri P, Lacava R, et al. Use of zolpidem in over 75-year-old patients with sleep disorders and comorbidities. Arch Gerontol Geriatr Suppl 2004;(9):93-6.

20. Scharf M, Erman M, Rosenberg R, et al. A 2-week efficacy and safety study of eszoplicone in elderly patients with primary insomnia. Sleep 2005;28:720-7.

2I. Curtis LH, Ostbye T, Sendersky V, et al. Inappropriate prescribing for elderly Americans in a large outpatient population. Arch Intern Med 2004; I64: I62I-5.

22. James SP, Mendelson WB. The use of trazodone as a hypnotic: a critical review. J Clin Psychiatry 2004;65:752-5.

23. Kaynak H, Kaynak D, Gozukirmizi E, et al. The effects of trazodone on sleep in patients treated with stimulant antidepressants. Sleep Med 2004;5:15-20.

24. Pandi-Perumal SR, Zisapel N, Srinivasan V, et al. Melatonin and sleep in aging population. Exp Gerontol 2005;40:9II-25.

25. Olde Rikkert MG, Rigaud AS. Melatonin in elderly patients with insomnia: a systematic review. Z Gerontol Geriatr 200I;34:49I-7.

26. Buscemi N, Vandermeer B, Hooton N, et al. The efficacy and safety of exogenous melatonin for primary sleep disorders: a meta-analysis. J Gen Intern Med 2005;20: II5I-8.

27. Buscemi N, Vandermeer B, Hooton N, et al. Efficacy and safety of exogenous melatonin for secondary sleep disorders and sleep disorders accompanying sleep restriction: meta-analysis. $B M J$ 2006;332:385-93

28. McGechan A, Wellington K. Ramelteon. CNS Drugs 2005;19:1057-65.

29. Fantini ML, Gagnon JF, Filipini D, et al. The effects of pramipexole in REM sleep behavior disorder. Neurology 2003;6I:I4I8-20.

30. Mignot E, Nishino S. Emerging therapies in narcolepsy-cataplexy. Sleep 2005; 28 : $754-63$. 
3I. US Xyrem Multicenter Study Group. Sodium oxybate demonstrates long-term efficacy for the treatment of cataplexy in patients with narcolepsy. Sleep Med 2004;5:119-23.

32. US Xyrem Multicenter Study Group. A I2-month, open-label, multicenter extension trial of orally administered sodium oxybate for the treatment of narcolepsy. Sleep 2003;26:31-5.

33. National Heart, Lung, and Blood Institute Working Group on Restless Legs Syndrome. Restless legs syndrome: detection and management in primary care. Am Fam Physician 2000;62:108-I4

34. Milligan SA, Chesson AL. Restless legs syndrome in the older adult: diagnosis and management. Drugs Aging 2002;19:74I-5I.

35. Comella CL. Restless legs syndrome: treatment with dopaminergic agents. Neurology 2002;58(Suppl I):S87-92.

36. Trenkwalder C, Hundemer HP, Lledo A, et al; PEARLS Study Group. Efficacy of pergolide in the treatment of restless legs syndrome: the PEARLS Study. Neurology 2004;62:139I-7

37. Schade R, Andersohn F, Suissa S, et al. Dopamine agonists and the risk of cardiacvalve regurgitation. $N$ Engl J Med 2007;356:29-38.

38. Zanettini R, Antonini A, Gatto G, et al. Valvular heart disease and the use of dopamine agonists for Parkinson's disease. N Engl JMed 2007;356:39-46.

39. Montplaisir J, Denesle R, Petit D. Pramipexole in the treatment of restless legs syndrome: a follow-up study. EurJ Neurol 2000;7(Suppl I):27-3I.

40. Bogan RK, Fry JM, Schmidt MH, et al; TREAT RLS US Study Group. Ropinirole in the treatment of patients with restless legs syndrome: a US-based randomized, double-blind, placebo-controlled clinical trial. Mayo Clin Proc 2006;81:17-27.

4I. Weimerskirch PR, Ernst ME. Newer dopamine agonists in the treatment of restless legs syndrome. Ann Pharmacother 2001;35:627-30.
42. Kyrmizakis DE, Chimona TS, Papadakis CE, et al. Laser-assisted uvulopalatoplasty for the treatment of snoring and mild obstructive sleep apnea syndrome. JOtolaryngol 2003;32:174-9.

43. Jones TM, Earis JE, Calverley PM, et al. Snoring surgery: a retrospective review. Laryngoscope 2005;115:2010-5.

44. Shochat T, Pillar G. Sleep apnoea in the older adult: pathophysiology, epidemiology, consequences and management. Drugs Aging 2003;20:551-60.

45. Quinnell TG, Smith IE. Obstructive sleep apnea in the elderly: recognition and management considerations. Drugs Aging 2004;21:307-22.

46. Montserrat JM, Ferrer M, Hernandez L, et al. Effectiveness of CPAP treatment in daytime function in sleep apnea syndrome: a randomized controlled study with an optimized placebo. Am J Respir Crit Care Med 200I;164:608-13.

47. Russo-Magno P, O'Brien A, Panciera T, et al. Compliance with CPAP therapy in older men with obstructive sleep apnea. J Am Geriatr Soc 200I;49:I205-II.

48. Mohsenin N, Mostofi MT, Mohsenin V. The role of oral appliances in treating obstructive sleep apnea. J Am Dent Assoc 2003;134:442-9.

49. Bradley TD, Logan AG, Kimoff RJ, et al; CANPAP Investigators. Continuous positive airway pressure for central sleep apnea and heart failure. $\mathrm{N}$ Engl J Med 2005; 353:2025-33.

Correspondence to: Dr. Norman Wolkove, Mount Sinai Hospital Center, 5690 Cavendish Blvd., Montréal QC $\mathrm{H}_{4} \mathrm{~W}_{\mathrm{I}} \mathrm{S}_{7}$; fax 5I4 369-2225; norluco@yahoo.com
Every year, we distribute more than 1.5 million copies of CMAJ to Canadian physicians and health professionals around the world.

\section{CMAJ}

for the best of Canadian medicine

Orders and information:

CMA Member Service Centre

tel $888855-2555$ or

$613731-8610 \times 2307$

fax $613236-8864$

cmamsc@cma.ca
Chaque année, nous distribuons de par le monde plus de 1,5 million d'exemplaires du $J A M C$ aux médecins et aux professionnels de la santé.

\section{Le JAMC}

tout simplement la crème de la médecine canadienne

Abonnements et demandes de renseignements :

Centre des services aux membres de l'AMC tél 888 855-2555 ou

$613731-8610 \times 2307$

fax $613236-8864$

cmamsc@cma.ca 